\title{
II. DoBles Y DOBLECES
}


